\title{
The Phylogenetic Roots of Addiction: Compulsive Drug Seeking, Natural and Drug-Sensitive Reward, and the Acquisition of Learned Habits
}

\author{
Robert Huber $^{\text {a }}$ Daniel A. Jacobson ${ }^{b, c}$ \\ a Department of Biological Sciences, Bowling Green State University, Bowling Green, OH, USA; b Biosciences Division,

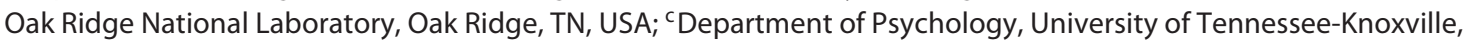 \\ Knoxville, TN, USA
}

Our rational faculties permit us humans to maximize the utility of our actions. We perform a fundamental type of cost-benefit analysis in which we frame a problem, assign values to the different paths, and then choose from among a set of available options, the course that promises the most favorable outcome. So why then does addiction appear to be so impervious to the associated costs, so unaffected by undesired consequences, and ultimately so resistant to cognitive oversight? The answer may likely be found in the fact that the drivers for compulsive drug seeking and drug taking are located in affective brain circuits, circuits that are structured and patterned by learning with repeated activation. These deep processes exhibit significant resistance to control by our cognitive faculties. The general consensus is that addiction arises from mechanisms that overvalue the magnitude of reward, discount the associated risks, and thereby bias individuals towards compulsive pursuit of addictive drugs. Behavioral disruption and dependence appear to arise at the intersection of a number of connected but separate phenomena: expectations for the occurrence of specific events, behaviors that seek encounters with them, the ability to notice and learn nonrandom, co-occurring conditions, the prediction and valuation of consequences, the

karger@karger.com

(c) 2021 S. Karger AG, Basel

www.karger.com/bbe

Karger forming of enduring memories, and the drivers of focused behavior through compulsion, habits, and acquired routines. It is important to recognize that each one of these individual faculties are present and well developed across the entire phylogenetic tree of bilateral metazoans. The goal of this special volume is dedicated to exploring the degree to which inherent elements can account for addiction and addiction-associated phenomena. In much of the literature on addiction, the underlying processes are often viewed as distinctly mammalian, arising, in part, from the strong cognitive capacities of this taxon. Some phenomena may even be regarded to exist only in primates, or even solely in humans. This supposition arises from the fact that studies are conducted almost exclusively in mammals and primates, while evolutionary antecedents of the behavior are rarely considered. A more comprehensive perspective that examines drug reward and reinforcement in a wider range of organisms demonstrates that many of the component traits are actually well developed across the greater metazoan lineage, and they may well predate the emergence of a mammalian clade by a wide margin.

The collection of papers assembled here supports the notion that the capacity to associate cues and conse- 
quences has not arisen in mammals de novo. Rather, the neural mechanisms for detecting contingencies and for predicting future outcomes are very deeply rooted across broad phylogenetic divisions. Our understanding of an ability to associate paired events has been enriched by work in invertebrate preparations in both classical and operant conditioning scenarios [Cook and Carew, 1986, 1989a, 1989b]. The ability to learn allows us to connect cues and behavioral actions to their associated consequences. Pavlovian conditioning enriches surrounding cues with predictive value. Outcomes with positive valence generate appetitive responses and approach to the associated cues, while those perceived as aversive bring cue avoidance and withdrawal. Humans are not the only life forms capable of such short- and long-term modulations of behavior.

Recognizing the presence of phylogenetic antecedents of addiction is more than just an academic exercise. A real benefit accrues from bringing a comparative phylogenetic perspective to bear on this phenomenon. We may garner new insights into its neural implementation, ontogeny and pathogenesis, relationship with other diseases and traits, behavioral expressions, and ultimately its ruinous consequences and destructive potential for the afflicted individual and society at large. By investigating the characteristics of organisms, including their life histories and behaviors, we are able to address the underlying rules that guide the evolution of addiction-associated phenomena and their neural implementations. With an eye on phylogenetic trajectories we gain an understanding of ancestral states, the timing of particular innovations, the tempo and mode of evolutionary change, and the underlying causes of correlations between traits and environments. Comparative frameworks, therefore, are a rich source of hypotheses in need of development and testing. Our ability to explore underlying commonalities permits us to link genetic, molecular, and neuronal implementations with behavioral expressions in intact animals. This approach tackles the broader roles played by neural mechanics in complex behavioral phenomena, such as addiction, natural and drug-sensitive reward, in incentive salience and persistent labelling of predictive cues, and in their underlying motivational drivers.

Addiction ultimately manifests as conspicuous expression of compulsive seeking and consumption despite the presence of adverse consequences. Key drivers reside in an altered expression of motivation and learning, individual capacities which can be traced back to at least the early precambrian [Vernier et al., 1995; Menzel and Benjamin, 2013]. The ability to learn arose early in animal evolution to provide adaptive and flexible behavioral responses using a limited complement of neuro-computational resources. In general, the evolutionary process has molded neural systems for solutions that maximize successful goal pursuit in an uncertain world. When a particular metabolic need is sensed, a drop in energy stores perhaps, neural controls generate perceptions of hunger. This state entices the individual to venture out and increases opportunity for encountering the resources essential to its survival. Such behavioral enhancers have been captured with many different terminologies, including motivations, drives, or the capacity to activate SEEKING, as one of Panksepp's seven Primary Emotions [Panksepp, 2004]. Psychostimulant drugs are thought to exert their effect by directly driving the underlying neural circuits responsible for these phenomena. The psychostimulant effects of invertebrates closely resemble those found in mammals for substituted phenethylamines [Alcaro et al., 2011; Huber et al., 2011; Gore et al., 2020] (i.e., amphetamines and cathinones) or alcohol [Swierzbinski et al., 2017]. Rewarding properties of psychostimulant drugs were confirmed in crayfish conditioned place preference experiments [Imeh-Nathaniel et al., 2016]. Robust bias for the associated sensory environment arose when it was paired with infusions of amphetamine, cocaine, methamphetamine, and opioids [Panksepp and Huber, 2004; Nathaniel et al., 2010; Imeh-Nathaniel et al., 2016]. Drug self-administration has been reported in roundworms with cocaine, nicotine, and methamphetamine [Engleman et al., 2018] and in crayfish with amphetamine [Datta et al., 2018]. Invertebrate models also exhibit strong tendencies for relapse after prolonged periods of abstinence [Nathaniel et al., 2009]. The proboscis extension reflex of honey bees was used to explore whether cocaine affects memory processing independently of its effect on incentive salience [Søvik et al., 2018]. Understanding how cocaine strongly impairs consolidation of extinction memory is key to understanding the enduring impacts of cocaine on behavior. Such research in invertebrate taxa traces the origins of addiction-like phenomena to at least 950 MYA [Dohrmann and Wörheide, 2017].

Placozoa, at a basal position in the metazoan phylogeny, coordinate sophisticated behavioral sequences, such as feeding, using an intercellular peptidergic signaling system [Varoqueaux et al., 2018]. This is the clearest indication yet that the revelatory power of phylogenetic perspectives has only just begun to infuse our understanding of the intricacies of addiction and addiction-like phenomena. 
The presence of exceedingly deep phylogenetic roots of addiction is supported by the observation that addictive pharmacologies arise from secondary plant metabolites acting as potent chemical defenses against insect herbivory [Wink, 2018]. Many are potent parasympathomimetics that stimulate monoamine neurotransmitter systems, in particular for dopamine [Eshleman et al., 2014; Pistillo et al., 2015]. The tradeoffs required for efficient learning - balancing specificity with generalization, and neuro-computational speed with behavioral flexibility - are key to an individual's ability for navigating life's demands. Evolutionary arms races have shaped the behavioral effects of nicotine, cathinone, ephedrine, morphine, and others into weaponized disruptors of critical neural machinery in insects. These compounds commandeer neural drivers for motivated search, they dysregulate reward perception, disrupt cue learning, and thereby interfere with the emergence of adaptive responses [Huber and van Staaden, 2019]. Human vulnerabilities may thus arise from the presence of conserved neural mechanisms that date back to early common ancestors. The use of the term "human drugs of abuse" for these compounds may thus be a misnomer. The ability to compare behavioral effects of addictive drugs across a broad range of taxa provides both a starting point for comparisons between taxa and perhaps telling implications for how we conceptualize, research, and treat addiction.

When we appreciate the presence of deep phylogenetic roots in addiction, we gain the use of powerful tools in experimentally tractable organisms, tools that allow us to disentangle the complex sets of mechanisms at play. Phenotypes themselves emerge from the complex interactions of genes in the context of life history and physiology, and individual phenotypic assays can accurately measure appropriately defined traits.

Moreover, new sophisticated approaches are required to explore the complex underlying genetic and omics architectures responsible for addiction phenotypes. Drosophila, for instance, offers an excellent model system to explore addiction and addiction-like phenomena. As an insect, it is within the original group of targets for plant secondary metabolites. Furthermore, Drosophila allows us to apply population-level phenotyping of complex behaviors shared with mouse [Baer et al., 2017; Angel et al., 2018] and human [Cirelli, 2009] and for studying human neural disorders including work relevant to substance abuse and toxicity [Grell et al., 1965; Gill et al., 1989; Muqit and Feany, 2002; Shulman et al., 2014]. Rich resources and tools exist from decades of research from the Drosophila research community [Popis et al., 2018], in- cluding recombinant inbred line (RIL) populations for GWAS mapping (DSPR [Long et al., 2014], DGRP [Mackay et al., 2012]), gene deletion collections (DrosDel [Ryder et al., 2004]), and extensive sets of omics data.

High-throughput behavioral phenotyping in RIL populations allows us to harness sophisticated network- and explainable-AI-based approaches [Joubert et al., 2018; Cliff etal., 2019] to discover epistaticinteractions (GWES). These modern computational biology tools offer a rich hypothesis generation engine to elucidate the complex mechanisms underlying addiction. In addition, multiplex networks can be constructed from the vast array of omics data publically available for Drosophila. These networks can be used with lines of evidence (LOE) algorithms in order to filter GWAS/GWES results to remove false positives and capture false negatives [Weighill et al., 2018, 2019; Furches et al., 2019; Chhetri et al., 2020]. The use of algorithms that can reveal interactions amongst genes and across defined phenotypes collectively offer strong predictors of addiction outcomes. In such capacity, they can provide extensive new insights into novel and complex molecular mechanisms. These methodologies for high-throughput hypothesis generation and filtering, combined with the ability to rapidly delete or overexpress genes or sets of genes in Drosophila with techniques such as CRISPR-Cas9 [Port et al., 2014; Lin et al., 2015; Port and Bullock, 2016; Jia et al., 2018], have the potential to yield an unparalleled discovery engine to thoroughly explore and manipulate the mechanisms underlying addiction. By recognizing the deep phylogenetic roots that exist in the causation of addition, we gain a rich set of experimental tools that offer novel insights and the explanatory frameworks with which to interpret them.

\section{Conflict of Interest Statement}

The authors have no conflicts of interest to declare.

\section{Funding Sources}

D.J. would like to thank the National Institute on Drug Abuse of the National Institutes of Health for funding support (R01DA051908 and R01DA051913]. This article has been co-authored by UT-Battelle, LLC under contract No. DE-AC0500OR22725 with the US Department of Energy. The United States Government retains and the publisher, by accepting the article for publication, acknowledges that the United States Government retains a nonexclusive, paid-up, irrevocable, world-wide license to publish or reproduce the published form of this article, or allow others to do so, for United States Government purposes. The De- 
partment of Energy will provide public access to these results of federally sponsored research in accordance with the DOE Public Access Plan (http://energy.gov/downloads/doe-public-accessplan, last accessed September 16, 2020).

\section{Author Contributions}

Both authors have contributed equally to the editorial.

\section{References}

Alcaro A, Panksepp J, Huber R. d-Amphetamine stimulates unconditioned exploration/approach behaviors in crayfish: towards a conserved evolutionary function of ancestral drug reward. Pharmacol Biochem Behav. 2011 Jul;99(1):75-80.

Angel C, Glovak ZT, Alami W, Mihalko S, Price J, Jiang Y, et al. Buprenorphine Depresses Respiratory Variability in Obese Mice with Altered Leptin Signaling. Anesthesiology. 2018 May;128(5):984-91.

Baer A, Bourdon AK, Spano G, Bellesi M, Bradley A, Tononi G, et al. Metabolomic Analysis of Microdialysis Samples from the Frontal Association Cortex (FrA) of C57BL/6J (B6) Mouse during Isoflurane Anesthesia. FASEB J. 2017;31(1 suppl).

Chhetri HB, Furches A, Macaya-Sanz D, Walker AR, Kainer D, Jones P, et al. Genome-Wide Association Study of Wood Anatomical and Morphological Traits in Populus trichocarpa. Front Plant Sci. 2020 Sep;11:545748.

Cirelli C. The genetic and molecular regulation of sleep: from fruit flies to humans. Nat Rev Neurosci. 2009 Aug;10(8):549-60.

Cliff A, Romero J, Kainer D, Walker A, Furches A, Jacobson D. A High-Performance Computing Implementation of Iterative Random Forest for the Creation of Predictive Expression Networks. Genes (Basel). 2019 Dec;10(12):E996.

Cook DG, Carew TJ. Operant conditioning of head waving in Aplysia. Proc Natl Acad Sci USA. 1986 Feb;83(4):1120-4.

Cook DG, Carew TJ. Operant conditioning of head-waving in Aplysia. III. Cellular analysis of possible reinforcement pathways. J Neurosci. 1989b Sep;9(9):3115-22.

Cook DG, Carew TJ. Operant conditioning of head-waving in Aplysia. II. Contingent modification of electromyographic activity in identified muscles. J Neurosci. 1989a Sep;9(9):3107-14.

Datta U, van Staaden M, Huber R. Crayfish SelfAdminister Amphetamine in a Spatially Contingent Task. Front Physiol. 2018 May;9:433.

Dohrmann M, Wörheide G. Dating early animal evolution using phylogenomic data. Sci Rep. 2017 Jun;7(1):3599.

Engleman EA, Steagall KB 2nd, Bredhold KE, Breach M, Kline HL, Bell RL, et al. Caenorhabditis elegans Show Preference for Stimulants and Potential as a Model Organism for Medications Screening. Front Physiol. 2018 Aug;9:1200.

Eshleman AJ, Forster MJ, Wolfrum KM, Johnson RA, Janowsky A, Gatch MB. Behavioral and neurochemical pharmacology of six psycho- active substituted phenethylamines: mouse locomotion, rat drug discrimination and in vitro receptor and transporter binding and function. Psychopharmacology (Berl). 2014 Mar;231(5):875-88.

Furches A, Kainer D, Weighill D, Large A, Jones P, Walker AM, et al. Finding New Cell Wall Regulatory Genes in Populus trichocarpa Using Multiple Lines of Evidence. Front Plant Sci. 2019 Oct; 10:1249.

Gill HJ, Nida DL, Dean DA, England MW, Jacobson KB. Resistance of Drosophila to cadmium: biochemical factors in resistant and sensitive strains. Toxicology. 1989 Jun;56(3):31521.

Gore S, van Staaden MJ, Sprague JE, Huber R. Synthetic cathinones and their phenethylamine analogues produce distinct psychomotor and reward behavior in crayfish. Behav Brain Res. 2020 Feb;379:112368.

Grell EH, Jacobson KB, Murphy JB. Alcohol Dehydrogenase in Drosophila melanogaster: Isozymes and Genetic Variants. Science. 1965 Jul;149(3679):80-2.

Huber R, Panksepp JB, Nathaniel T, Alcaro A, Panksepp J. Drug-sensitive reward in crayfish: an invertebrate model system for the study of SEEKING, reward, addiction, and withdrawal. Neurosci Biobehav Rev. 2011 Oct;35(9):1847-53.

Huber R, van Staaden M, editors. Invertebrate Models of Natural and Drug-Sensitive Reward. Frontiers Media (SA); 2019. https://doi. org/10.3389/978-2-88945-928-5.

Imeh-Nathaniel A, Adedeji A, Huber R, Nathaniel TI. The rewarding properties of methamphetamine in an invertebrate model of drug addiction. Physiol Behav. 2016 Jan;153:40-6.

Jia Y, Xu RG, Ren X, Ewen-Campen B, Rajakumar $R$, Zirin J, et al. Next-generation CRISPR/ Cas9 transcriptional activation in Drosophila using flySAM. Proc Natl Acad Sci USA. 2018 May;115(18):4719-24.

Joubert W, Weighill D, Kainer D, Climer S, Justice A, Fagnan K, et al. Attacking the Opioid Epidemic: Determining the Epistatic and Pleiotropic Genetic Architectures for Chronic Pain and Opioid Addiction. In: SC18: International Conference for High Performance Computing, Networking, Storage and Analysis. 2018. pp. 717-730. https://doi. org/10.1109/SC.2018.00060.

Lin S, Ewen-Campen B, Ni X, Housden BE, Perrimon N. In Vivo Transcriptional Activation Using CRISPR/Cas9 in Drosophila. Genetics. 2015 Oct;201(2):433-42.

Long AD, Macdonald SJ, King EG. Dissecting complex traits using the Drosophila Synthetic
Population Resource. Trends Genet. 2014 Nov;30(11):488-95.

Mackay TF, Richards S, Stone EA, Barbadilla A, Ayroles JF, Zhu D, et al. The Drosophila melanogaster Genetic Reference Panel. Nature. 2012 Feb;482(7384):173-8.

Menzel R, Benjamin PR. Chapter 1 - Beyond the Cellular Alphabet of Learning and Memory in Invertebrates. In: Menzel R, Benjamin PR. Invertebrate Learning and Memory. Handbook of Behavioral Neuroscience. Elsevier Inc.; 2013, vol 22. pp. 3-5.

Muqit MM, Feany MB. Modelling neurodegenerative diseases in Drosophila: a fruitful approach?NatRevNeurosci.2002Mar;3(3):23743.

Nathaniel TI, Panksepp J, Huber R. Drug-seeking behavior in an invertebrate system: evidence of morphine-induced reward, extinction and reinstatement in crayfish. Behav Brain Res. 2009 Feb;197(2):331-8.

Nathaniel TI, Panksepp J, Huber R. Effects of a single and repeated morphine treatment on conditioned and unconditioned behavioral sensitization in Crayfish. Behav Brain Res. 2010 Mar;207(2):310-20.

Panksepp J. Affective Neuroscience: The Foundations of Human and Animal Emotions. Oxford: Oxford University Press; 2004.

Panksepp JB, Huber R. Ethological analyses of crayfish behavior: a new invertebrate system for measuring the rewarding properties of psychostimulants. Behav Brain Res. 2004 Aug;153(1):171-80.

Pistillo F, Clementi F, Zoli M, Gotti C. Nicotinic, glutamatergic and dopaminergic synaptic transmission and plasticity in the mesocorticolimbic system: focus on nicotine effects. Prog Neurobiol. 2015 Jan;124:1-27.

Popis M, Borowiec B, Jankowski M. Drosophila Melanogaster Research: History, Breakthrough and Perspectives. Med J Cell Biol. 2018. https://doi.org/10.2478/acb-2018-0028.

Port F, Bullock SL. Augmenting CRISPR applications in Drosophila with tRNA-flanked sgRNAs. Nat Methods. 2016 Oct;13(10):8524.

Port F, Chen HM, Lee T, Bullock SL. Optimized CRISPR/Cas tools for efficient germline and somatic genome engineering in Drosophila. Proc Natl Acad Sci USA. 2014 Jul;111(29):E2967-76.

Ryder E, Blows F, Ashburner M, Bautista-Llacer $\mathrm{R}$, Coulson D, Drummond J, et al. The DrosDel collection: a set of P-element insertions for generating custom chromosomal aberrations in Drosophila melanogaster. Genetics. 2004 Jun;167(2):797-813. 
Shulman JM, Imboywa S, Giagtzoglou N, Powers MP, Hu Y, Devenport D, et al. Functional screening in Drosophila identifies Alzheimer's disease susceptibility genes and implicates Tau-mediated mechanisms. Hum Mol Genet. 2014 Feb;23(4):870-7.

Søvik E, Berthier P, Klare WP, Helliwell P, Buckle EL, Plath JA, et al. Cocaine Directly Impairs Memory Extinction and Alters Brain DNA Methylation Dynamics in Honey Bees. Front Physiol. 2018 Feb;9(February):79.
Swierzbinski ME, Lazarchik AR, Herberholz J. Prior Social Experience Affects the Behavioral and Neural Responses to Acute Alcohol in Juvenile Crayfish. J Exp Biol. 2017 Apr;220(Pt 8):1516-23.

Varoqueaux F, Williams EA, Grandemange S, Truscello L, Kamm K, Schierwater B, et al. High Cell Diversity and Complex Peptidergic Signaling Underlie Placozoan Behavior. Curr Biol. 2018 Nov;28(21):3495-3501.e2.

Vernier P, Cardinaud B, Valdenaire O, Philippe $\mathrm{H}$, Vincent JD. An evolutionary view of drugreceptor interaction: the bioamine receptor family. Trends Pharmacol Sci. 1995 Nov;16(11):375-81.
Weighill D, Jones P, Bleker C, Ranjan P, Shah M, Zhao N, et al. Multi-Phenotype Association Decomposition: Unraveling Complex GenePhenotype Relationships. Front Genet. 2019 May;10(May):417.

Weighill D, Jones P, Shah M, Ranjan P, Muchero W, Schmutz J, et al. Pleiotropic and Epistatic Network-Based Discovery: Integrated Networks for Target Gene Discovery. Front Energy Res. 2018;6:30.

Wink M. Plant Secondary Metabolites Modulate Insect Behavior-Steps Toward Addiction? Front Physiol. 2018 Apr;9:364. 